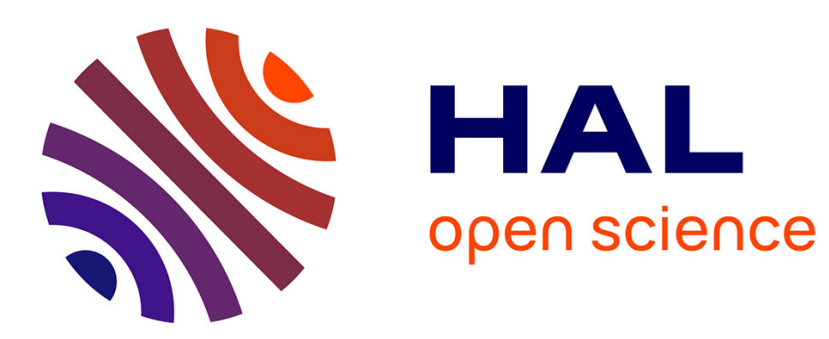

\title{
Une action publique sans coopération politique: Le style languedocien de la politique régionale
}

\author{
Emmanuel Négrier
}

\section{To cite this version:}

Emmanuel Négrier. Une action publique sans coopération politique: Le style languedocien de la politique régionale. Pôle Sud - Revue de science politique de l'Europe méridionale, 1998, Elections et politiques régionales, 8, pp.41-53. 10.3406/pole.1998.993 . hal-02484103

\section{HAL Id: hal-02484103 \\ https://hal.umontpellier.fr/hal-02484103}

Submitted on 22 Apr 2020

HAL is a multi-disciplinary open access archive for the deposit and dissemination of scientific research documents, whether they are published or not. The documents may come from teaching and research institutions in France or abroad, or from public or private research centers.
L'archive ouverte pluridisciplinaire HAL, est destinée au dépôt et à la diffusion de documents scientifiques de niveau recherche, publiés ou non, émanant des établissements d'enseignement et de recherche français ou étrangers, des laboratoires publics ou privés.

\section{(ㅇ)(1) $\$$}

Distributed under a Creative Commons Attribution - NonCommercial - NoDerivatives $\mid 4.0$ 


\section{Une action publique sans coopération politique : Le style} languedocien de la politique régionale

Mr Emmanuel Négrier

\section{Citer ce document / Cite this document :}

Négrier Emmanuel. Une action publique sans coopération politique : Le style languedocien de la politique régionale. In: Pôle Sud, $n^{\circ} 8,1998$. Elections et politiques régionales. pp. 41-53;

doi : https://doi.org/10.3406/pole.1998.993

https://www.persee.fr/doc/pole_1262-1676_1998_num_8_1_993

Fichier pdf généré le 23/04/2018 


\title{
Une action publique sans coopération politique : Le style languedocien de la politique régionale.
}

\author{
par Emmanuel Négrier \\ CEPEL-CNRS
}

Pôle Sud $N^{\circ} 8-$ mai $1998-p .41$ à 53.

\begin{abstract}
Cet article se donne pour objectif d'analyser la manière dont la région met en ceuvre ces capacités d'intervention et compétences dans le cadre de dix années d'action publique territoriale. Il repose sur une série de travaux réalisés dans plusieurs domaines des politiques régionales en Languedoc-Roussillon. Plus que de retracer de manière exhaustive dix années de politique régionale, il essaie d'en tirer les enseignements qui paraissent essentiels pour situer la Région dans le concert territorial, national et européen d'action publique. C'est la raison pour laquelle nous privilégions trois développements thématiques : les effets de la contractualisation de l'action régionale en Languedoc-Roussillon; l'analyse de deux domaines choisis pour leur exemplarité (culture et recherche), et la régionalisation des politiques européennes.
\end{abstract}

Avec un budget de près de 3 milliards de francs en 1997, soit le dixième rang national, et le huitième en francs par habitant, la région Languedoc-Roussillon fait figure de région française moyenne. La dernière des collectivités territoriales en date est aussi celle qui a vu sa capacité d'action financière augmenter le plus rapidement ces dix dernières années, au fur et à mesure que s'étoffaient les Contrats de Plan État-région, la fiscalité propre du Conseil régional et sa politique d'emprunt. La ventilation de ses dépenses fait apparaître le poids de l'éducation et de la formation (environ $46 \%$ du budget, dont $601 \mathrm{MF}$ pour la formation professionnelle et $593 \mathrm{MF}$ pour les lycées). Ceci traduit une forte augmentation des investissements en matière d'infrastructures scolaires, même si le Languedoc-Roussillon se place au douzième rang français pour la dépense par habitant. Le développement économique (347 MF) et l'aménagement du territoire (334 MF) se situent au second rang. Viennent ensuite l'ensemble sportculture-loisirs (108 MF) et l'environnement (74 MF).

Les ressources du Conseil régional proviennent, pour 1,5 milliard de francs, de la fiscalité, pour 853 millions de francs des dotations et participations (financement national et européen notamment), et pour 340 millions de francs de l'emprunt. De ce point de vue, la région LanguedocRoussillon est parmi les plus endettées (en nombre d'années nécessaires pour rembourser la dette, selon les chiffres de l'Expansion $n^{\circ} 566$, février 1998) et est la septième des régions qui prélèvent le plus d'impôt par habitant.

Ladministration régionale dispose, pour mettre en œuvre ses politiques, d'un personnel qui s'est considérablement élargi, pour atteindre aujourd'hui environ 250 personnes, ce qui correspond à la moyenne 


\section{Pôle Sud $N^{\circ} 8$}

nationale du nombre d'agents par habitant. Ces agents se répartissent de la façon suivante : $33 \%$ de cadre A, $11 \%$ de cadres $B$ et $56 \%$ de cadres $C$ (le plus fort taux français hors DOM-TOM et Corse). La proportion de fonctionnaires dans cette administration est de $84,5 \%$, soit le plus fort taux national (devant Rhône-Alpes : $79,5 \%$ et la Corse : $79 \%)$. La fonction publique territoriale $(82 \%)$ y occupe également le premier rang en France, tandis que les fonctionnaires d'État $(2,5 \%)$ sont parmi les plus minoritaires.

Si la région dispose ainsi des moyens humains et financiers pour mener à bien de véritables politiques territoriales, il n'en demeure pas moins vrai que la régionalisation de l'action publique en France est inachevée. Pour autant, à contraintes juridiques et économiques égales, les Conseils Régionaux ont d'ores et déjà démontré des styles de politique et des capacités d'intervention très contrastées, et ce dans des domaines aussi différents que l'aménagement du territoire, la mise en œuvre des politiques européennes, les politiques culturelles ou de recherche.

Cet article se donne pour objectif d'analyser la manière dont la région met en œuvre ces capacités d'intervention et compétences dans le cadre de dix années d'action publique territoriale. Il repose sur une série de travaux réalisés dans plusieurs domaines des politiques régionales en Languedoc-Roussillon. Nous n'avons pas pour ambition de retracer ici de manière exhaustive dix années de politique régionale, mais plutôt d'en tirer les enseignements qui nous paraissent essentiels pour la situer dans le concert territorial, national et européen d'action publique. C'est la raison pour laquelle nous allons privilégier trois développements : les effets de la contractualisation de l'action régionale en Languedoc-Roussillon; deux domaines choisis pour leur exemplarité (culture et recherche), et la régionalisation des politiques européennes.

\section{Une politique sous influence contractuelle?}

La politique régionale a pour première caractéristique d'être prise dans la logique de la contractualisation ${ }^{1}$. Celle-ci, qui passe par l'instrument des Contrats de Plan ÉtatRégion, vient compenser les effets de la liberté que l'on attribue, comparativement aux autres collectivités, au Conseil régional. S'il n'a que peu de dépenses obligatoires à satisfaire, et donc une marge de manœuvre plus importante sur ses investissements, il est de plus en plus contraint d'assumer une nouvelle forme d'interdépendance par le biais des Contrats de Plan. Ceci étant, la manière dont les régions gèrent cette interdépendance diffère sensiblement d'une région à l'autre. En Languedoc-Roussillon, plusieurs éléments importants sont à noter.

Tout d'abord, cette région s'illustre par une des plus faibles parts françaises de cofinancement territorial, qui concerne la contribution à ces contrats des autres collectivités territoriales. Entre 1989 et 1993 , la part de l'État est de 2,7 milliards de francs, celle de la région de 1,4 et celle des autres collectivités locales de 0,04 . Si, dans la deuxième phase de programmation (1994-1998), cette part augmente (818 millions de francs pour 1,5 milliard de part 


\section{Une action publique sans coopération politique}

régionale et 3,6 pour l'État), c'est essentiellement dû aux effets de l'incorporation, dans la douleur, du Plan Université 2000. Cette participation des autres niveaux territoriaux est très largement supérieure dans la plupart des autres régions, pour s'établir en moyenne à un rapport de $45 \% / 55 \%$. On peut y voir un premier indice de l'extrême difficulté pour la Région de mobiliser autour d'elle un réel partenariat territorial.

Ensuite, on note que la part des compétences éducatives dans le co-financement État-région est très élevée, largement audessus des autres pratiques régionales. L'État en finance $65 \%$, au lieu de $50 \%$ voire moins pour les autres régions. Il est par contre bien moins présent sur les autres volets, plus volontaristes ou "libres" de la politique régionale : culture, sport, aménagement du territoire... Ceci confirme, du côté de l'État cette fois, les limites de la coopération institutionnelle : la région ne parvient à l'engager que sur les domaines où elle est contrainte à la mise en œuvre de ses compétences : recherche, éducation, financements européens. Encore n'y est-elle pas en position nécessairement dominante. On le verra plus loin pour l'action européenne. On peut d'ores et déjà le souligner pour l'enseignement supérieur et la recherche : contrainte à intégrer, après bien des résistances de l'exécutif régional, le plan Université 2000, le Conseil a été conduit, pour faire valoir ses propres priorités, à s'engager seul sur beaucoup d'actions qui n'avaient pas l'aval de l'État (certaines antennes universitaires ou para-universitaires, publiques et privées). Il y a ainsi pesé plus que dans un contexte partenarial, mais sur des volumes d'action plus faibles, s'interdisant de fait d'influer sur les domaines les plus structurants ou essentiels : l'enseignement supérieur à Montpellier (notamment l'Université de Montpellier II, pourtant pôle incontournable de sa stratégie de Multipôle Technologique Régional, $c f$. infra) a été considéré comme terra non grata. Puisque cette ville concentrait $80 \%$ du potentiel régional, l'idée était de faire porter l'effort sur d'autres sites, par ailleurs plus en phase avec ses propres orientations politiques. En dehors de circonstances liées à l'urgence (L'Université Paul Valéry de Lettres, aux prises avec des problèmes dramatiques de locaux) ou à une opportunité de marquage politique (la rénovation de la Faculté de Droit de Montpellier). Le LanguedocRoussillon est la région française qui compte le plus de chantiers de cet ordre où les partenaires officiels (du CPER) se sont de fait répartis les cartes de telle manière qu'à chaque fois un seul soit en charge de la totalité de la responsabilité.

Enfin, au contraire là aussi de bien d'autres régions, le Conseil régional du Languedoc-Roussillon a davantage suivi la contractualisation qu'il n'a tenté de peser dessus pour en faire un instrument de son autonomisation : les grands chapitres des Contrats ont été laissés tels quels, sans tentative de reformulation ou de passerelles entre secteurs (exemple : formation professionnelle et agriculture, développement local et industriel, recherche et formation,...). La région s'est inscrite dans à peu près toutes les actions initiées par l'État (la DATAR par exemple avec sa politique des Pactes Territoriaux pour l'Emploi), sans 


\section{Pôle Sud $N^{\circ} 8$}

réelle sélectivité de son intervention ni possibilité d'enrôler en retour l'État et les autres collectivités dans des politiques qu'elle aurait elle-même définies.

D'une certaine manière, cette stratégie a eu pour conséquence de libérer le Conseil régional d'une étreinte trop forte de ses partenaires naturels. L'absence quasi-générale de partenariat sur les opérations horscontrat signifie pour le Conseil la possibilité de décider seul de l'affectation des ressources. Elle peut se comprendre ainsi comme la volonté de structurer de véritables clientèles politiques. Le grand nombre d'organismes financés par la région dans le cadre de la politique de formation professionnelle est un indice manifeste de cette tendance au saupoudrage, malgré l'introduction de dispositifs d'encadrement (par branches : métallurgie, transport, industrie hôtelière, automobile, bâtiment; ou par l'introduction de chartes de qualité). Les caractéristiques de cette politique régionale, outre la précocité de sa prise en charge par le Conseil régional, dès juillet 1994 (soit la première région à s'inscrire dans ce cadre de décentralisation issu de la loi quinquennale de 1993), sont, d'après le Comité National de Coordination des Programmes Régionaux d'Apprentissage et de Formation Professionnelle Continue, au nombre de deux. La région LanguedocRoussillon est parmi celles qui, confrontées à un fort taux de chômage, ont eu le plus recours à des dispositifs locaux et spécifiques vis-à-vis de ceux de l'État. Elle est aussi celle où la présence de l'État est des plus marquées, avec une sur-représentation des dispositifs de formation alternée et d'apprentissage.
Mais l'absence de coopération sur les domaines hors-contrat est aussi le signe d'une incapacité notable à orienter les autres acteurs dans le cadre d'une politique régionale propre, cohérente et originale visà-vis de celle de l'État. Incapacité qui n'est pas imputable seulement à la politique de l'exécutif régional (malgré la forte personnalisation de la politique régionale depuis 12 ans), mais aussi aux partenaires (départements, villes) qui ont déserté eux-mêmes la scène régionale.

\section{Deux cas de politique régionale}

Nous avons choisi de porter l'accent sur deux cas de figure symboliques de l'action régionale : la culture, la recherche. Outre qu'ils sont au cœur des débats en cours sur le devenir de la régionalisation et sur les implications possibles de la présence au sein de la majorité d'un parti extrémiste, ils sont des révélateurs des formes par lesquelles une institution régionale peut peser sur l'action publique, et du style particulier que celle-ci revêt en Languedoc-Roussillon.

\section{La politique culturelle régionale}

La politique régionale en matière culturelle suit une double logique. Elle est d'une part, comme toutes les autres régions, liée à la politique de l'État par le biais de la contractualisation. C'est à la faveur de celleci qu'ont été lancés les FRAC (Fonds Régional d'Art Contemporain) et FRAM (Fonds Régional d'Acquisition pour les Musées) et qu'a pu s'opérer une bonne partie des actions de la Région en matière d'équipements et de développement culturel. Cette dépendance mutuelle ne signifie 


\section{Une action publique sans coopération politique}

pas que la coopération entre l'État et la région soit sans conflit, et que les nouvelles compétences ainsi acquises par le Conseil régional au fil des contrats aient conduit à un modèle stable de politique culturelle. Au contraire, à l'image de ce qui s'est produit depuis les débuts des FRAC, ces deux partenaires ont, face aux ressortissants (artistes, galeristes, experts,...), une attitude ambiguë à l'égard de toute action commune. Les conflits portent tant sur la politisation supposée des choix artistiques qui, au moins jusqu'à aujourd'hui, n'a pas trouvé de confirmation réelle, que sur la suprématie de critères d'excellence artistique ayant peu à voir avec le territoire régional (Four, 1993). L'une des orientations principales de la Région aura été, en cette matière, de soutenir une création régionale qui disposait, dans certains secteurs, de lettres de noblesse (le courant Support-Surface par exemple). Ce soutien n'a que très tardivement conduit (en 1997) au financement d'un lieu spécifique d'exposition. Enfin, face à une Direction Régionale des Affaires Culturelles qui dispose d'experts nombreux et reconnus et, depuis 1992, d'une autonomie plus marquée à l'égard de la préfecture, le Conseil régional n'a pas développé de capacité équivalente, de nature à lui permettre de peser significativement sur les orientations culturelles régionales. Bien au contraire, la dissolution de toute structure collective au niveau régional (de type Office ou Comité régional) a conduit à une individualisation des relations entre l'institution et un milieu fourni (l'un des plus denses des régions françaises en créateurs et en emplois culturels) déjà marqué par un fort individualisme.
À côté de cette première dimension contractuelle, la région a développé une politique de créneau plus étroite. Là où elle était plus libre de ses investissements, et en dehors d'une stratégie de subventionnement très éclatée entre genres, disciplines et secteurs, la région a privilégié deux domaines : la culture occitane d'une part, et la politique du livre de l'autre. Cette stratégie s'explique d'abord par le fait qu'il s'agit de domaines qui identifient fortement la région. La culture occitane, par ses traditions et sa pratique contemporaine, et le livre, par la densité de ses éditeurs (l'une des toutes premières de France) ont représenté dès 1986 un symbole fort de politique régionale. Leur identification plus volontiers à gauche (pour les représentants de ces secteurs et une bonne partie des acteurs concernés par l'intervention régionale) n'était pas sans intérêt pour un exécutif issu d'une alliance avec le Front National. Le découplage qui s'opère ici entre la culture occitane et les traditions de mobilisations occitanes, aux antipodes du conservatisme politique, est un des acquis de cette stratégie. Concernant les éditeurs, la labellisation du Centre Régional des Lettres, à partir de ce qui était une simple association d'intérêts, suit la même logique culturelle et politique, en conférant une autonomie assez large à l'organisme (Négrier, 1994), qui lui sera utile lorsque J. Blanc confiera au Front National les responsabilités sur la culture au sein du Conseil régional, en 1986.

La logique de créneau se comprend ainsi doublement. Elle consacre une tentative d'enrôlement d'intérêts préalablement structurés sur le territoire, auxquels elle confere légitimité institutionnelle, ressources humaines et financières et autonomie orga- 


\section{Pôle Sud $N^{\circ} 8$}

nisationnelle (Baraize, Négrier, 1997). De ce point de vue, le CRL est une réussite incontestable sur ces trois plans, bien qu'elle ne conduise qu'à une coopération inter-institutionnelle marginale avec l'État, et que ses orientations "sectorielles" redoublent plus qu'elles ne dépassent, sur le plan territorial, la fracture nationale entre politique du Livre et politique de la lecture (Surel, 1997; De La Salle, 1996). Elle consacre aussi une stratégie de compétition politique à au moins deux entrées. Comme on l'a vu, le choix des créneaux n'est pas indifférent à la nature politique des milieux concernés. Le soutien à la culture occitane a ainsi pu capitaliser les soutiens d'artistes qui, pour être plus volontiers classés à gauche, s'étaient sentis victimes des inflexions "professionnalisantes" des politiques culturelles des villes, et singulièrement de celle de Montpellier (Négrier, 1993). En se saisissant de ces opportunités, le Conseil Régional a pluralisé une offre publique culturelle auprès des artistes, qui était déjà elle-même fragmentée entre villes et Conseils Généraux.

Le second aspect de cette stratégie concerne les politiques d'image déployées par le Conseil régional dans l'ordre événementiel (un festival de musique, l'organisation de Jeux Méditerranéens...), pour certains directement concurrents de ceux de la ville de Montpellier, aux mêmes périodes. Pour avoir connu un succès beaucoup plus douteux, ces entreprises n'en sont pas moins significatives d'une compétition entre institutions qui, eu égard à la personnalisation de leurs exécutifs, a rendu centrale la question des allégeances politico-personnelles à l'un ou l'autre des leaders régionaux. Elle a également conduit à une impossibilité logique : celle du financement commun de structures pourtant d'envergure régionale (l'Orchestre Philarmonique, le festival de Radio-France, le Corum-Palais des Congrès de Montpellier - Négrier, 1996).

\section{La politique régionale de la recherche}

La recherche occupe une place importante dans l'activité régionale. 10.000 personnes employées dans des équipes de recherche, ce qui représente le cinquième taux régional le plus important en France, tant vis-à-vis de la population totale, que vis-à-vis de la valeur ajoutée régionale. Les trois-quarts de ces effectifs publics et privés sont situés à Montpellier. En 1990, le budget annuel de la recherche était de 450 millions de francs (hors CEA2). Le LanguedocRoussillon est la cinquième région française pour ce qui concerne le nombre de thèses soutenues, quatrième pour le montant de crédits par chercheur, sixième pour la production d'articles scientifiques, septième pour la part des crédits provenant du Fonds de la Recherche et de la Technologie (FRT) et des conventions CIFRE (entrepriseslaboratoires). Elle est cependant au douzième rang seulement pour ce qui concerne le dépôt de brevets, ce qui peut être un indice d'un assez grand déséquilibre entre l'offre publique de recherche et de ressources en transfert de technologie, abondante, et la faible capacité d'innovation du tissu industriel, naissant, lui-même. C'est dans ce domaine que le Conseil régional a fait porter un effort particulier à travers le dispositif générique de "Multipôle Technologique Régional". 


\section{Une action publique sans coopération politique}

\section{Budget de fonctionnement des Pôles (en MF)}

\begin{tabular}{lrrrrrrrrr}
\hline Pôle & \multicolumn{1}{c}{$\begin{array}{c}\mathbf{1 9 9 4} \\
\text { Région }\end{array}$} & Total & État & $\begin{array}{c}1995 \\
\text { Région }\end{array}$ & Total & État & $\begin{array}{l}1996 \\
\text { Région }\end{array}$ & Total \\
\hline Productique & 350 & 350 & $\mathbf{7 0 0}$ & 200 & 450 & $\mathbf{6 5 0}$ & 350 & 450 & 800 \\
IA2 & 500 & 400 & $\mathbf{9 0 0}$ & 500 & 500 & $\mathbf{1 0 0 0}$ & 400 & 500 & $\mathbf{9 0 0}$ \\
Membrane & 500 & 500 & $\mathbf{1 0 0 0}$ & 500 & 500 & 1000 & 500 & 500 & $\mathbf{1 0 0 0}$ \\
Verseau & 500 & 350 & $\mathbf{8 5 0}$ & 500 & 500 & $\mathbf{1 0 0 0}$ & 500 & 500 & $\mathbf{1 0 0 0}$ \\
TRIAL & 500 & 400 & $\mathbf{9 0 0}$ & 500 & 800 & $\mathbf{1 3 0 0}$ & 800 & 800 & $\mathbf{1 6 0 0}$ \\
Construction & 250 & 200 & $\mathbf{4 5 0}$ & 200 & 200 & $\mathbf{4 0 0}$ & 200 & 500 & $\mathbf{7 0 0}$ \\
GBM & 0 & 0 & $\mathbf{0}$ & 0 & 0 & $\mathbf{0}$ & 0 & 0 & $\mathbf{0}$ \\
Carnot & 0 & 0 & $\mathbf{0}$ & 100 & 143 & $\mathbf{2 4 3}$ & 0 & 125 & 125 \\
Total & 2600 & 2600 & $\mathbf{5 2 0 0}$ & 2500 & 3093 & 5443 & 2750 & 3575 & $\mathbf{6 3 2 5}$ \\
\hline
\end{tabular}

Source : Evaluation du MTR (CESR, 1997)

Les Pôles Technologiques Régionaux constituent le mode d'intégration régionale privilégié de ces dispositifs. Ils sont au nombre de huit : CARNOT (systèmes et procédés énergétiques), CONSTRUCTION, GBM (génie biologique et médical), IA2 (Intelligence artificielle et informatique appliquée), PRODUCTIQUE, MEMBRANES, TRIAL (Industrie Agroalimentaire), VERSEAU (sciences et techniques de l'eau). Leur budget est alimenté par des subventions de l'État et de la région, ainsi que par des contrats industriels. Ils sont intégrés au MTR et leur mission s'étend depuis l'animation et l'interface recherche-industrie jusqu'à la constitution de réels programmes de recherche-développement. Le IX' Plan (1984-1988) avait vu se réaliser 470 projets industriels, dont 170 en recherche-développement, pour environ 300 entreprises régionales.

La politique régionale en la matière est essentiellement articulée sur le MTR, dont la logique fédérative repose sur trois objectifs officiels :
- favoriser le développement de la recherche en région;

- réaliser le transfert technologique et l'appui technique pour les entreprises régionales;

- promouvoir l'aménagement du territoire dans le domaine de la recherche et de la technologie.

Son budget de fonctionnement pour 1996 était de 3,5 millions de Francs.

L'évaluation du dispositif MTR fait apparaitre les points suivants :

- les deux instruments (Pôles et CLAT) disposent d'une présence territoriale (pour les premières) et sectorielle (pour les secondes) assez homogène, bénéficient d'une autonomie qui leur permet de mener à bien des stratégies de coopération avec les acteurs institutionnels locaux et régionaux, tout en étant souvent proches des intérêts économiques et scientifiques. Leur capacité de mobilisation, sur des secteurs représentatifs de l'économie régionale, s'appuie généralement sur des conseillers techniques professionnels. 


\section{Pôle Sud $N^{\circ} 8$}

- les conditions de coopération interinstitutionnelles demeurent problématiques pour plusieurs raisons : manque de volonté réciproque de coopération avec les CCI, inadaptation du statut des Cellules pour des missions trop éclatées, défaut de reconnaissance par les acteurs les plus pertinents et turn-over très important des conseillers techniques, lourdeur des tâches administratives qui absorbent une partie des activités opérationnelles, et trésorerie fragile.

Le dispositif régional apparaît donc morcelé, sans pilotage centralisé. La multiplicité des pilotes partiels, celle des objectifs assignés aux différentes entités, et enfin celle des structures juridiques adoptées conduisent à une très grande imprécision du dispositif lui-même. Le réseau MTR, qui n'a pas de limite précise mais semble au contraire se déployer au gré des opportunités, pêche par son manque de visibilité externe, mais également de communication entre ses différentes composantes. Son incription dans le réseau plus large des acteurs publics économiques (DRIRE, CCI, Départements et Villes) demeure problématique après plus de dix ans d'existence.

Le cadre régional, comme dans d'autres domaines, n'a pas le monopole de l'encadrement de la recherche et du transfert de technologie. La présence, sur ce champ, des collectivités départementales mais surtout des villes, à travers les technopoles, repose davantage sur une logique de compétition (symbolique tout autant que pratique) que de coopération (Michel, 1993).

\section{La région, niveau discuté d'action publique européenne}

Il est devenu banal d'affirmer que les politiques européennes ont représenté, pour les régions, un instrument de légitimation précoce et notable dans l'action publique territoriale. Encore faut-il en mesurer, au-delà des discours, les modalités concrètes et styles particuliers, qui different là encore d'une région à l'autre L'étude de la mise en ouvre régionale des politiques européenne en Languedoc-Roussillon, à laquelle nous venons de consacrer un rapport (CEPEL, 1998 ; Négrier-Jouve, 1998), nous conduit à quatre constats principaux :

- elle est moins régionaliste qu'il n'y paraît de premier abord. Si la réforme des fonds structurels de 1988 a ouvert une fenêtre d'opportunité à la mobilisation régionale, elle a également mis en éveil d'autres niveaux d'action, que l'on croyait parfois en déclin (le département), ou en perte de légitimité (L'État central). Si l'on a cru pouvoir déceler, dans cette réforme qui conduisait à insister sur le partenariat territorial, une stratégie délibérée de la Commission Européenne pour favoriser le niveau "régional", force est d'en mesurer aujourd'hui les effets. La région est devenue certes un partenaire incontournable de l'action publique territoriale, mais elle est loin d'être le seul, et souvent loin d'être en mesure d'en être le chef de file incontesté. Cela ne signifie d'ailleurs pas que le "retour de l'État", dont on a fait, à partir de 1994, un thème majeur lors des inflexions postérieures à la première phase de programmation (1989-1993), soit si évident. La reprise en main de certains aspects de la 


\section{Une action publique sans coopération politique}

programmation n'a en aucune manière donné à l'État (en région) les ressources nécessaires pour sortir du partenariat, et renoncer (pour le meilleur et pour le pire) au compromis territorialisé.

- la subsidiarité, qui est à partir de la ratification du Traité de Maastricht un peu plus qu'une idée social-chrétienne pour devenir un principe normatif, a bien du mal à rentrer dans les mœurs françaises en général, languedociennes en particulier. Certains jugeront que ce principe n'est applicable qu'aux États fédéraux ou fédéralisants, où le compromis, le consensus sont ancrés dans une culture et élevés au rang d'un art qui nous resteraient radicalement étrangers. C'est mettre trop de contenu normatif dans un principe qui, au contraire, est une invite à négocier, à débattre, plus qu’à figer des répartitions de compétences. La subsidiarité existe dans les politiques européennes régionales. Elle s'est simplement traduite en LanguedocRoussillon, comme dans d'autres régions, par des dispositifs à géométrie variable. Il n'y a pas d'ordre subsidiaire intangible, parce qu'existent une pluralité de compétences et des acteurs à la légitimité partielle et variable selon les programmes concernés. C'est en ce sens qu'en lieu et place d'acteurs institutionnels subsidiaires et permanents, on a pu voir peser ici un département, ici un réseau d'experts, là la région et l'État dont, en son intérieur, les diverses composantes locales ne sont pas toujours unifiées et sans conflits.

- du fait de ces variations dans l'ordre subsidiaire des compétences et des légitimités, l'action publique européenne en région est rendue plus sensible à la mobilisation d'intérêts institutionnels et sociaux spécifiques. On a pu le voir dans les dispositifs de reconversion industrielle (bassin d'Alès) ou de développement rural dans l'arrière-pays languedocien, où les "bons vieux acteurs du territoire" disposent d'une capacité d'influence notable. Celle-ci se traduit non seulement par le fait qu'ils obtiennent la satisfaction de leurs sollicitations financières, mais, et peut-être surtout, qu'ils sont parfois dominants dans le travail, inévitable, de traduction concrète des programmes. On le sait, les cadres officiels d'action européenne (les DOCUP) sont complexes, fournis, souvent inaccessibles à des acteurs pourtant rodés aux financements public-privé. Leur traduction en opérations concrètes et formellement compatibles apparaît comme une véritable activité politique. Elle a pour corollaire la confirmation du rôle pivot d'institutions (exemple : le Conseil général dans le PDZR, et dans le programme LEADER; la DRIRE et la sous-préfecture dans la reconversion industrielle alésienne). C'est pour cela que l'on a parfois l'impression que les politiques européennes innovent peu, que la visibilité de l'intervention structurelle se noie dans des programmes cofinancés, où les contreparties nationales et locales, souvent redondantes, viennent soutenir des politiques traditionnelles.

- pour paradoxal que cela puisse paraître, les politiques européennes ont cependant conduit à une mobilisation considérable d'acteurs régionaux. Que celle-ci soit souvent le fruit d'une stimulation institutionnelle (les pouvoirs locaux en appelant à leurs réseaux territoriaux pour encadrer les programmes) ne change rien au fait que, sur une période de 10 ans, les fonds structurels soient désormais connus, les dispositifs reconnus et les opportunités qu'ils offrent rendus attractifs. 


\section{Pôle Sud $N^{\circ} 8$}

A travers cinq études de cas, nous avons ainsi pu voir émerger de nouveaux pôles, sinon de pouvoir, du moins de compétence, dont on peut attribuer l'existence, au moins en partie, à l'Europe. Il en est ainsi de la ville d'Alès dans le développement économique, des réseaux d'experts dans le patrimoine rural ou dans le développement technologique, et, bien sûr, du Conseil régional, notamment dans l'inter-régional. Cet apprentissage élargi, par le biais de l'Europe, de ce que recouvre l'action partenariale, modifiera à terme les conditions de mise en œuvre des politiques régionales. On est aujourd'hui forcé de constater que l'élargissement n'est pas maximal, loin s'en faut. Des bastions demeurent, des exclusions se confirment (comme celle de l'insertion par l'économique, à peu de choses près, dans la reconversion industrielle),... bref l'intégration sociale, promue par les injonctions communautaires, reste limitée par les effets de leadership. La comparaison entre le LanguedocRoussillon et les autres régions du panel REGE (la Catalogne, l'Andalousie, le Paysde-Galles, le Bade-Wurtemberg, la BasseSaxe, La Lombardie et la Sicile) montre d'ailleurs que la différence de perception entre les acteurs régionaux directement impliqués dans les programmes communautaires et les autres est maximale. Ceci traduit bien cet écart entre des "acteurs régionaux européens" et une opinion publique régionale qui a montré, dans les sondages, mais surtout dans son vote à l'occasion de la ratification du Traité de Maastricht, sa réticence à l'égard de l'intégration européenne. Les fonds structurels, sur les espaces (notamment ruraux) où ils étaient largement distribués, n'ont pas contribué, de ce point de vue, à la diffusion d'un sentiment pro-européen dans la population régionale. Mais l'apprentissage est de longue haleine, et le leadership ne se reproduit qu'en se renouvelant...

La région Languedoc-Roussillon, comme bien d'autres, a, dès la première phase de sa jeune existence démocratique, tenté de fonder sa légitimité sur le contournement des autres institutions territoriales, à commencer par l'État. Compte tenu de la fragmentation politique et du sens du conflit inter-institutionnel, cela s'est traduit par une compétition presque généralisée sur les créneaux que la région s'ouvrait (culture, relations interrégionales, ressources européennes, développement économique,...). Parallèlement à cette première stratégie, l'exécutif régional a développé ses propres relais territoriaux, dont le meilleur exemple est sans doute l'appui, pour le développement économique, sur le réseau des CCI. Ceci était lié à des logiques sectorielles, mais reposait bien sûr aussi sur des alliances politiques. En tentant d'enrôler politiquement des fractions, ou des individualités participant de l'action ou des forces politiques régionales, le Conseil régional s'est constitué en acteur lui-même, au risque d'une certaine forme de clientélisme. Cette double stratégie (peser par l'extérieur, tisser des réseaux individualisés sur le territoire) n'a pas pourtant conduit à une claire identification de l'action régionale comme telle. La première a donné naissance à des structures ou partenariats souvent limités (Euro-région, Communauté de Travail des Pyrénées, AREV,...), qui ne constituent encore que des "plates-formes virtuelles" d'action publique. La seconde a plus confirmé les équilibres ou fragmentations politiques existants qu'elle n'a contribué à les réorienter dans le cadre d'une 


\section{Une action publique sans coopération politique}

véritable régionalisation de l'action publique. Elle a ainsi laissé un champ d'action à l'État que l'on ne retrouve pas forcément dans d'autres régions.

La période qui s'ouvre aujourd'hui, avec la remise en cause d'un droit garanti aux fonds structurels et la vraisemblable généralisation de logiques de projet, renforce l'importance des facteurs qualitatifs mis en évidence plus haut. La remise en question de la politique régionale, due à l'élargissement de l'Union européenne, devrait aller de pair avec le relatif effacement des Programmes d'Intérêt Communautaire, sauf dans les domaines transfrontalier et inter-régional. La concentration de cette politique régionale, sur trois objectifs au lieu de six actuellement, constituera une importante pression à une initiative régionale structurée sur l'innovation et le projet. Plus que sur les logiques plus ou moins standardisées de programmation, les régions (et les autres niveaux d'action territoriale) devront désormais compter sur leurs propres capacités d'échange politique pour demeurer les interlocuteurs légitimes de l'action européenne. Cela suppose, de la part des régions, et en Languedoc-Roussillon en particulier, une réflexion approfondie sur le partenariat inter-institutionnel. Elle concerne d'abord des choix stratégiques, où le fait de privilégier le contournement des autres acteurs légitimes peut s'avérer encore plus pénalisant demain qu'hier. Elle se rapporte enfin aux moyens, qui ne sont aujourd'hui plus négligeables, dont dispose le Conseil Régional pour peser sur des orientations territoriales à la définition desquelles il est désormais associé depuis plus de dix ans.
Les instruments dont dispose la région Languedoc-Roussillon ont beau demeurer largement inférieurs en moyens à ceux des autres collectivités territoriales, ils ont donné naissance à des formes d'intervention qui ne peuvent plus aujourd'hui être négligées. Si l'évaluation de leur utilisation laisse entrevoir un certain nombre de limites générales à la régionalisation en France, elle permet aussi de qualifier celles qui sont directement imputables à une stratégie politique particulière et, plus généralement, à un positionnement de la région dans les dynamiques politiques languedociennes. Au risque d'être schématique, on peut définir trois composantes majeures de l'action régionale en Languedoc-Roussillon :

- elle est fort peu partenariale. Au moment où la notion de partenariat s'enrichit et laisse apparaître de substantielles différences entre régions ( $c f$. l'article de P. Duran dans ce numéro), il est significatif de constater combien sont grands les obstacles à la coopération inter-institutionnelle, tant avec l'État lui-même qu'avec les autres collectivités.

- elle est considérablement marquée par une compétition politique vive et de tous les instants qui privilégient l'affrontement à la gestion collective comme mode d'identification politique pour la plupart des acteurs territoriaux. Très politique dans sa version "personnalisée" du leadership, cette compétition est en même temps très peu politique au sens collectif du terme.

- elle empêche, dans la plupart des domaines où la politique régionale pouvait y conduire, à une orientation régionale de s'imposer au-delà de la fragmentation des scènes d'action publique. De ce point de vue, 


\section{Pôle Sud $N^{\circ} 8$}

loin d'être le chef de file ou, au moins, un niveau particulier d'impulsion et de régulation de l'action publique, le Conseil Régional apparaît comme l'un des espaces, parmi d'autres, d'allocation parcellaire de ressources publiques.

En conséquence, l'un des traits de cette action, parfois souligné au long de ce bref article, est son caractère clientéliste et personnalisé. L'imbrication des logiques politiques, d'intérêts organisés ou individuels et des domaines d'intervention en constituent la première condition. La fragmentation des réseaux et l'individualisation des soutiens en est la seconde. La manière dont J. Blanc a obtenu puis élargi sa majorité régionale en 1986, et l'a conservée en 1992 est typique de cette économie des ralliements individuels ${ }^{3}$. Tout se passe comme si le Conseil régional, à travers ses politiques, s'était constitué en une sorte de bloc de ressources patrimoniales, assez personnalisé, dont l'équilibre tient à une accumulation d'allégeances individuelles et à la diabolisation d'un adversaire unique. Cette stratégie est bien sûr rendue praticable par l'attitude qu'observe depuis plus de dix ans l'opposition au sein du Conseil régional. À la faveur de la représentation proportionnelle, celle-ci y dispose d'une place privilégiée pour discuter publiquement les choix qui se sont opérés en LanguedocRoussillon. L'absence de débat provoqué par ceux-ci, la stratégie de défection ou d'abstention que l'opposition a généralement adoptée (un exemple parmi d'autres : le fait de laisser voter en bloc les chapitres budgétaires en Commission Permanente, ce qui empêchait tout contrôle réel des subventions individualisées) ont rendu cette "patrimonialisation" de l'action publique régionale possible. Elle n'a fait que renforcer les tendances au conflit et à l'évitement de la coopération. La période qui s'ouvre aujourd'hui va, au vu des nouvelles orientations européennes et de l'évolution de la politique contractuelle avec l'État, plutôt dans un sens inverse à ces tendances, qui consacrent un relatif isolement politique (en dehors même de circonstances politiques qui lui donnent un autre relief) du Conseil régional.

1. J'adresse ici mes remerciements à François Baraize, qui m'a permis, à l'occasion de nombreuses discussions, de nourrir cette réflexion de ses propres analyses et données recueillies à l'occasion d'un travail de thèse en cours d'achèvement sur les politiques universitaires.

2. Commissariat à l'Énergie Atomique

3. Dans le contexte actuel de la majorité UDF-RPR-FN, cette logique d'enrôlement individuel connaît toutefois de sérieuses limites, comme le montre l'insertion, dans le programme présidentiel du candidat Blanc, d'une partie de la doctrine exigée par le Front National, ou encore l'équilibre des forces droiteextrême droite, nettement plus favorable à cette dernière qu'auparavant. 


\section{Une action publique sans coopération politique}

\section{Références}

Baraize F., Négrier E., "Livres et écrits dans l'Europe des Régions " Rapport pour le Centre Régional des Lettres Languedoc-Roussillon, Programme PACTE de l'Union Européenne, CRL Montpellier, 1997.

De La Salle M., L'impuissance publique. La politique de la lecture publique en France (1945-1993), Thèse pour le doctorat de science politique (Paris I), 1996.

Four P.-A., " La compétence contre la décentralisation ? Création et re-création des Fonds Régionaux d'Art Contemporain ", Politix, $n^{\circ} 24,1993$.

Michel C., Le Multipôle Technologique Régional du Languedoc-Roussillon : entre décentralisation et transfert de technologie ", Mémoire de DEA de Science Politique (Montpellier I), 1993.

Négrier E. (dir.) : "Politique régionale et action publique européenne en Languedoc-Roussillon" Rapport pour le CNRS et le Conseil Régional Languedoc-Roussillon, Montpellier CEPEL, 1998.

Négrier E., "Languedoc-Roussillon : le Centre Régional des Lettres ", in Alliès P., Roche F., Négrier E., Pratiques des échanges culturels internationaux : les collectivités territoriales, Paris, La Documentation Française, 1994.

Négrier E., "Montpellier : international competition and community access " in Bianchini F., Parkinson M. (eds.), Cultural policy and urban regeneration. The West European Experience, Manchester, Manchester University Press, 1993.

Négrier E., "Une manifestation musicale au miroir de la ville. Le festival de Radio-France et (de) Montpellier ", in Darré A. (dir.), Musiques et politique. Les répertoires de l'identité, Rennes, Presses Universitaires de Rennes, 1996.

Négrier E., Jouve B. (dir.), Que gouvernent les régions d'Europe? Echanges politiques et mobilisations régionales, Paris, L'Harmattan, 1998.

Surel Y., L'État et le livre. Les politiques publiques du livre en France (1957-1993), Paris, L'Harmattan, 1997. 